\title{
MINIMUM DISTORTION LOOK-UP TABLE BASED DATA HIDING
}

\author{
Xiaofeng Wang, Xiao-Ping Zhang, Senior Member, IEEE \\ Department of Electrical \& Computer Engineering, \\ Ryerson University, Toronto, ON, Canada, M5B 2K3. \\ E-mail : xzhang@ee.ryerson.ca
}

\begin{abstract}
In this paper, we present a novel data hiding scheme based on the minimum distortion look-up table (LUT) embedding that achieves good distortion-robustness performance. We first analyze the distortion introduced by LUT embedding and formulate its relationship with run constraints of LUT. Subsequently, a Viterbi algorithm is presented to find the minimum distortion LUT. Theoretical analysis and numerical results show that the new LUT design achieves not only less distortion but also more robustness than the traditional LUT based data embedding schemes.
\end{abstract}

\section{INTRODUCTION}

Data hiding system plays a more and more important role in multimedia applications with the development of internet. Quantization based embedding [1] and spread-spectrum [2] are two most commonly used data hiding schemes. Quantization based method has the advantage of host interference rejecting. Look-up table (LUT) embedding is a simple and efficient quantization based scheme. The most popular LUT is odd-even embedding or dither modulation [1] which is LUT with run (number of consecutive zeros or ones) of 1. A pixeldomain LUT embedding scheme is proposed in [3], where the LUT is associated with a cryptographic key to provide security and it is a $n$-run LUT, i.e., the maximum allowable run of the LUT is $n$. Wu [4] indicated that $n$-run LUT embedding generally introduces larger distortion than the traditional odd-even embedding with the same quantization step size.

In this paper, we show that with the knowledge of the watermark data the LUT can be designed to achieve less distortion than the traditional schemes. From the analysis of the mean squared distortion introduced by $n$-run LUT, we find that the distortion can be greatly reduced by designing the LUT according to the distribution of the host data and the watermark data. We further formulate the minimization of the LUT distortion as a dynamic programming problem. A practical minimum-distortion $n$-run LUT design method is presented based on a Viterbi algorithm. Experiment results show that at the same watermark-to-noise ratio (WNR), the bit error rate (BER) for minimum-distortion $n$-run LUT embedding can be smaller than the odd-even embedding.

\section{OVERVIEW OF THE LUT EMBEDDING}

A LUT $T$ is a sequence of 0's and 1's. The table is associated with a uniform quantizer. It maps every feature or pixel value of an image to the quantization level according to the input data to be embedded. For example to embed a " 1 " in a pixel, the pixel is rounded by its quantization value if the entry of the table corresponding to that pixel is also a " 1 ". If the entry is not " 1 ", we should find its nearest quantization level for which its LUT entry is " 1 " to replace the pixel as illustrated in Fig. 1. The process of embedding " 0 " is the same. The look-

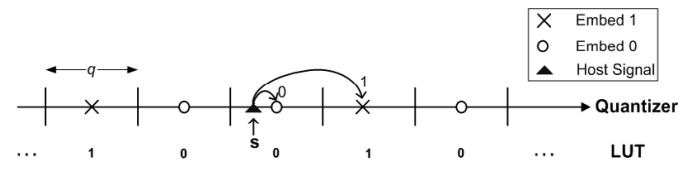

Fig. 1. An example of of LUT based data hidding

up function $\operatorname{Lookup}(\cdot)$ simply returns a "0" or " 1 " depending upon the input index,

$$
\operatorname{Lookup}(I)=\text { value in Look-up table at index } I \text {. }
$$

The $L U T(\cdot)$ function takes the value of the original signal as the input and maps it to a " 0 " or " 1 " according to the LUT. Thus, the $\operatorname{LUT}(\cdot)$ function is actually a simple composition of the lookup and the quantization functions:

$$
\operatorname{LUT}(s)=\operatorname{Lookup}(Q(s) / q),
$$

where $q$ is quantization step and $Q$ is quantization function .

The entire process altering a pixel can be abstracted into the following formula:

$$
x= \begin{cases}Q(s) & \text { if } \operatorname{LUT}(s)=b, \\ s+\delta & \text { if } \operatorname{LUT}(s) \neq b,\end{cases}
$$

where $s$ is the original feature, $x$ is the watermarked feature, $b$ is the bit to be embedded; and $\delta=\arg \min _{d}\{|d| \mid d=$ $(Q \operatorname{uant}(x)-s)$ s.t. $\operatorname{LUT}(x)=b\}$.

Once the LUT is known, the watermark detection can be easily implemented through a simple lookup from the LUT. The table is looked up as

$$
\hat{b}=\operatorname{LUT}(\hat{x}),
$$


where $\hat{b}$ is the extracted bit and $\hat{x}$ is the watermarked, possibly corrupted signal. It is desired to design the LUT such that the distortion is as small as possible.

\section{DISTORTION ANALYSIS}

In LUT embedding, uniform quantization $Q(\cdot)$ divides the input signal space into $K$ levels. If the $k$-th entry of LUT is $b$, to embed $b$ the data samples of signal $s$ in the quantization cell of $[(k-1 / 2) q,(k+1 / 2) q]$ is rounded to $k q$, the mean square distortion produced by this operation is calculated as

$$
D_{k}\left(s_{b}\right)=\int_{(k-1 / 2) q}^{(k+1 / 2) q}|s-k q|^{2} f(s) d s,
$$

where $f(s)$ is the Probability Density Function (PDF) of $s$ and the features to embed bit " $b$ " is denoted by $s_{b}$. However, if the bit to be embedded for $s$ is not $b$, the host data must be mapped to the nearest quantization point corresponding to the desired bit. There are three cases as illustrated in Fig. 2.

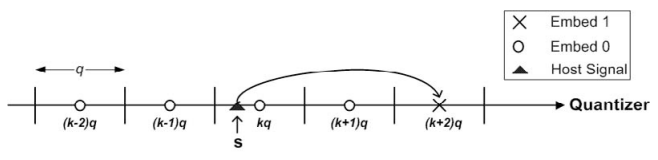

(a)

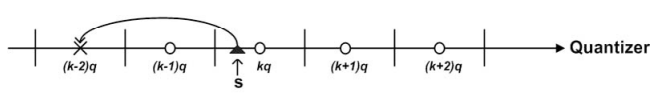

(b)

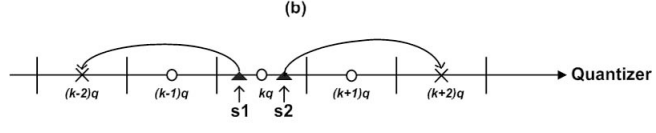

(c)

Fig. 2. Illustration of distortion analysis of embeding 1. (a) $(k+2)$-th entry is the only one closest entry. (b) $(k-2)$-th entry is the only one closest entry. (c) $(k+2)$-th and $(k-2)$-th entry are both closest entries.

If the $(k+l)$-th entry is the only one closest entry for the desired bit (Fig. 2(a)), the distortion of the $k$-th entry is

$$
\begin{aligned}
D_{k}^{+l}= & \int_{(k-1 / 2) q}^{(k+1 / 2) q}|s-(k+l) q|^{2} f(s) d s \\
= & D_{k}\left(s_{b}\right)+l^{2} q^{2} \int_{(k-1 / 2) q}^{(k+1 / 2) q} f(s) d s \\
& -2 l q \int_{(k-1 / 2) q}^{(k+1 / 2) q}(s-k q) f(s) d s .
\end{aligned}
$$

If the feature is approximately symmetric distributed within each cell, the last term is close to 0 . We have

$$
D_{k}^{+l} \approx D_{k}\left(s_{b}\right)+l^{2} q^{2} \int_{(k-1 / 2) q}^{(k+1 / 2) q} f(s) d s .
$$

Similarly, if the $(k-l)$-th entry is the only one closest entry for the desired bit (Fig. 2(b)), the distortion is

$$
D_{k}^{-l} \approx D_{k}\left(s_{b}\right)+l^{2} q^{2} \int_{(k-1 / 2) q}^{(k+1 / 2) q} f(s) d s .
$$

In another case, we have two nearest quantization points $(k+l) q$ and $(k-l) q$ corresponding to the desired bit simultaneously (Fig. 2(c)), then the original features in the range of $[(k-1 / 2) q, k q]$ will be rounded to $(k-l) q$, and the features in the other half interval $[k q,(k+1 / 2) q]$ will be mapped to $(k+l) q$. The distortion will be composed by two parts:

$$
\begin{aligned}
D_{k}^{ \pm l}= & \int_{(k-1 / 2) q}^{k q}|s-(k-l) q|^{2} f(s) d s \\
& +\int_{k q}^{(k+1 / 2) q}|s-(k+l) q|^{2} f(s) d s .
\end{aligned}
$$

Similarly it comes to

$D_{k}^{ \pm l} \approx D_{k}\left(s_{b}\right)+l^{2} q^{2} \int_{(k-1 / 2) q}^{(k+1 / 2) q} f(s) d s-\frac{l q^{2}}{2} \int_{(k-1 / 2) q}^{(k+1 / 2) q} f(s) d s$.

For a binary data hiding system, we can divide the features into two categories: the features that are used to embed bit " 0 ", denoted by $s_{0}$, and the features to embed bit " 1 ", denoted by $s_{1}$. The PDFs of $s_{0}$ and $s_{1}$ are $f_{0}\left(s_{0}\right)$ and $f_{1}\left(s_{1}\right)$, respectively. Now consider that each of the $K$ LUT entries is either " 0 " or " 1 ". In all $K$ quantization cells, all the data have to be mapped to the closest reconstruction points for the desired entry. According to (5) - (10), the overall $n$-run LUT $T$ embedding distortion for the feature within the $k$-th quantization cell can be formulated as

$$
\begin{aligned}
\operatorname{Dist}_{k}(T)= & D_{k}\left(s_{0}\right)+q^{2} \sum_{l=1}^{n-1}\left[l^{2} \alpha_{0, k}^{l}-\frac{l}{2} \beta_{0, k}^{l}\right] \\
& \cdot \int_{(k-1 / 2) q}^{(k+1 / 2) q} f\left(s_{0}\right) d s_{0} \\
& +D_{k}\left(s_{1}\right)+q^{2} \sum_{l=1}^{n-1}\left[l^{2} \alpha_{1, k}^{l}-\frac{l}{2} \beta_{1, k}^{l}\right] \\
& \cdot \int_{(k-1 / 2) q}^{(k+1 / 2) q} f\left(s_{1}\right) d s_{1},
\end{aligned}
$$

where $T$ is LUT, $\alpha$ and $\beta$ are calculated as follows:

$$
\begin{gathered}
\alpha_{0, k}^{l}=\max \left\{\overline{t_{k-l}} t_{k-l+1} . . t_{k} . . t_{k+l-1}, t_{k-l+1} . . t_{k} . . t_{k+l-1} \overline{t_{k+l}}\right\}, \\
\alpha_{1, k}^{l}=\max \left\{t_{k-l} \overline{t_{k-l+1}} . . \overline{t_{k}} . . \overline{t_{k+l-1}}, \overline{t_{k-l+1}} \cdot . \overline{t_{k}} . . \overline{t_{k+l-1}} t_{k+l}\right\}, \\
\beta_{0, k}^{l}=\alpha_{0, k}^{l} \overline{t_{k-l}} \overline{t_{k+l}}, \\
\beta_{1, k}^{l}=\alpha_{1, k}^{l} t_{k-l} t_{k+l},
\end{gathered}
$$

where $t$ is entry of T. $\alpha_{b, k}^{l}=1$ only when $t_{k} \neq b, t_{k-l}$ or $t_{k+l}$ or both is the nearest LUT entry for $b . \beta_{b, k}^{l}=1$ only when $t_{k} \neq b, t_{k-l}$ and $t_{k+l}$ are the nearest LUT entries for $b$. 
From (11), we can formulate the overall distortion as

$$
M S E_{w}(T)=\sum_{k=0}^{K-1} \operatorname{Dist}_{k}(T) .
$$

Considering the overall mean squared distortion due to quantization only is $M S E_{\text {quan }}=\sum_{k=0}^{K-1}\left[D_{k}\left(s_{0}\right)+D_{k}\left(s_{1}\right)\right]$. Let the additional distortion by data hiding at the $k$-th cell $D_{k}(T)=q^{2} \sum_{l=1}^{n-1}\left[l^{2} \alpha_{0, k}^{l}-\frac{l}{2} \beta_{0, k}^{l}\right] \int_{(k-1 / 2) q}^{(k+1 / 2) q} f\left(s_{0}\right) d s_{0}$ $+q^{2} \sum_{l=1}^{n-1}\left[l^{2} \alpha_{1, k}^{l}-\frac{l}{2} \beta_{1, k}^{l}\right] \int_{(k-1 / 2) q}^{(k+1 / 2) q} f\left(s_{1}\right) d s_{1}$.

We can rewrite the overall embedding distortion as

$$
M S E_{w}(T)=M S E_{\text {quan }}+\sum_{k=0}^{K-1} D_{k}(T) .
$$

\section{MINIMUM DISTORTION LUT WITH VITERBI ALGORITHM (VA)}

The overall structure of the proposed data hiding scheme is illustrated in Fig. 3 for binary case. The channel could be any

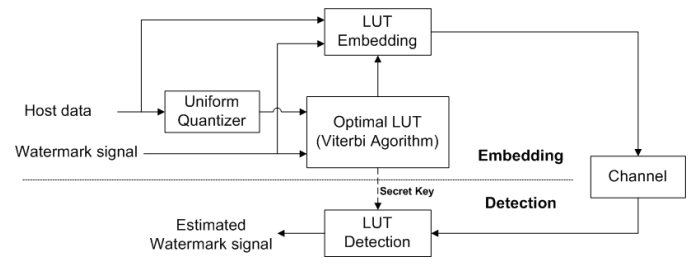

Fig. 3. Watermark Embedding and Detection Process

attack or noise. First host data are quantized using uniform quantizer. Then a Viterbi algorithm is used to find the optimal distortion LUT which is described below. After that the LUT is used as secret key to both embedding and detection sides.

Since $M S E_{\text {quan }}$ is the same for every $T$, the $n$-run LUT we want is the one that minimize the additional distortion,

$$
T_{\text {opt }}=\arg \min _{T}\left\{\sum_{k=0}^{K-1} D_{k}(T)\right\} .
$$

We formulate it as a problem of minimizing $K$ steps summation of $D_{k}(T)$ and it can be solved using dynamic programming. A Viterbi algorithm (VA) [5] is used. For a $n$-run LUT, we have $2^{2 n-2}$ states represented by the $2 n-2$ neighboring LUT entries:

$$
S=" t_{k-n+1} \ldots t_{k} \ldots t_{k+n-2} " .
$$

In each state of the trellis, the previous state metric (SM) and the corresponding branch metric (BM) are added together, and then the accumulated SM is updated by choosing the minimum of all possible cases recursively

$$
S M_{S_{i}}^{k+1}=\min _{S_{j}}\left(S M_{S_{j}}^{k}+B M_{S_{j}, S_{i}}^{k+1}\right), k=0, \ldots, K-2,
$$

where $S M_{S_{j}}^{k}$ represents the SM of the $j^{t h}$ state at step $k$, and $B M_{S_{j}, S_{i}}^{k}$ denotes the BM at step $k$ associated with a transition from state $S_{j}$ to state $S_{i}$. A transition happens only when the last $2 n-3$ entries of $S_{j}$ is the same as the first $2 n-3$ entries of $S_{i}$. Fig. 4 shows the trellis of a 2-run LUT.

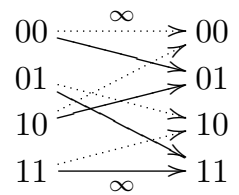

Fig. 4. Trellis of 2-run LUT. A arc is traversed if the next entry is 1 , a dotted arc is traversed if the next entry is 0 . Because the run is 2, the BM from "00" to "00" and from "11" to "11" is $\infty$.

The initial state metric $S M_{S_{i}}^{0}$ is given by the additional distortion of $k=0$.

$$
S M_{S_{i}}^{0}=D_{0}\left(S_{i}\right)
$$

where $D_{k}\left(S_{i}\right)$ denotes the additional distortion while $t_{k-n+1}, \cdots$ $t_{k}, \cdots, t_{k+n-1}$ are given by $S_{i}$.

Let $B M_{S_{j}, S_{i}}^{k}$ be the additional distortion of the $k$-th entry. Since the additional distortion of the $k$-th entry is decided only by the $2 \mathrm{n}-1$ nearby entries $t_{k-n+1}, \ldots, t_{k}, \ldots, t_{k+n-1}$ which can be obtained from $S_{j}$ and $S_{i}$. Considering the case that from $S_{j}$ to $S_{i}$ will break the run $n$ constrain, $B M_{S_{j}, S_{i}}^{k}$ is given by a modification of the additional distortion.

$$
B M_{S_{j}, S_{i}}^{k}=\left\{\begin{array}{lc}
\infty & \text { if } r u n>n \text { from } S_{j} \text { to } S_{i}, \\
D_{k}\left(S_{j}, S_{i}\right) & \text { else, }
\end{array}\right.
$$

where $D_{k}\left(S_{j}, S_{i}\right)$ denotes the additional distortion while $t_{k-n+1}, \cdots, t_{k}, \cdots, t_{k+n-1}$ are given by $S_{j}$ and $S_{i}$. Then the accumulated SM is the overall additional distortion for all the $K$ entries. Once the accumulated SM, and the history of the states the smallest accumulated SM is built up, we can create the minimum-distortion $n$-run LUT.

\section{EXPERIMENTAL RESULTS WITH IMAGES}

To verify our scheme, we apply our proposed minimum distortion LUT embedding with run constraint of 2 and 3 to the popular $512 \times 512$ images Lena, Bridge and Goldhill. One bit is embedded in each pixel. For comparison, an embedding scheme using the odd-even LUT was also implemented.

The following results are using Lena as an example and other pictures get the similar results. Fig. 5 is the PSNR (peak-signal-to-noise-ratio) after embedding of two schemes. As we expected, PSNR of the minimum distortion LUT embedding is better than the odd-even LUT embedding at all levels. When the number of quantization level increases, the 
difference between the two gets smaller. The underlying reason is that the distortion of the odd-even LUT embedding gets smaller with more quantization levels and leaves less space for improvement. That the 3-run LUT only has slight improvement over 2-run is due to the distribution of the watermark signal which is not far from uniform.

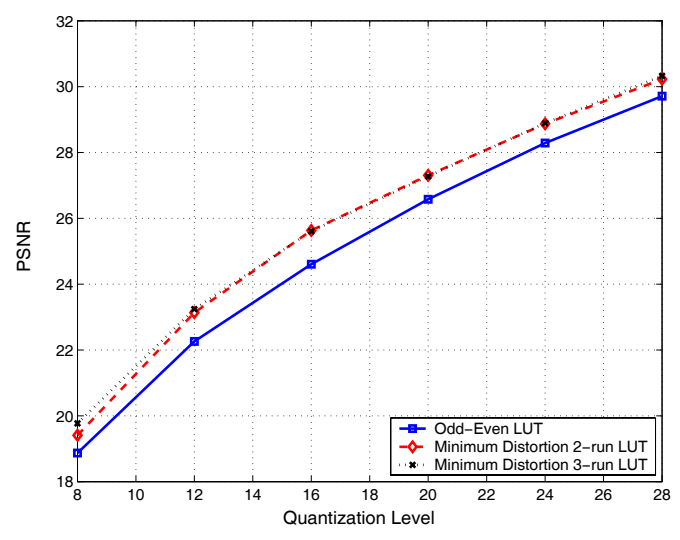

Fig. 5. PSNRs at different quantization levels for the minimum distortion and odd-even LUT.

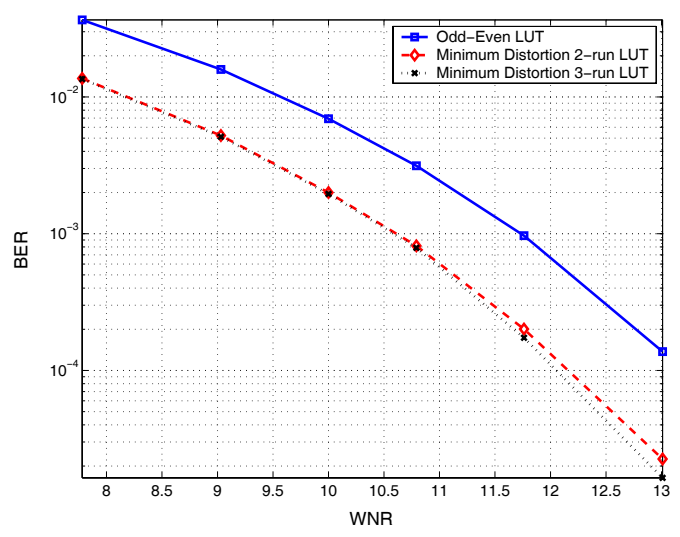

Fig. 6. BER versus WNR for the minimum distortion and odd-even LUT under white Gaussian noise. The quantization level is 20 .

Next, we add white Gaussian noise to watermarked images with the minimum distortion LUT and the odd-even LUT. The detection errors on $512 \times 512$-bit raw data at different WNR are shown in Fig. 6. Fig. 7 visualizes the detection errors from which we can see minimum distortion LUT has a great improvement on reducing the raw BER. And the PSNR is also increased from $26.54 \mathrm{~dB}$ of odd-even to $27.30 \mathrm{~dB}$ of minimum distortion LUT.

\section{CONCLUSION}

A new LUT data hiding scheme that minimizes distortion was designed. Through the distortion analysis, we generalized the
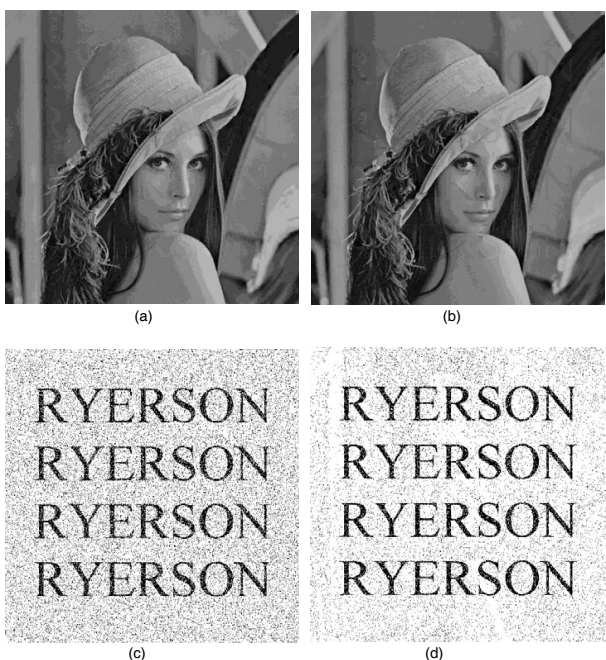

Fig. 7. The embedded image and raw error pattern with the odd-even LUT (a)(c) and the minimum distortion 3-run LUT (b)(d) under WNR $=4.77 \mathrm{~dB}$. The quantization level is 20 .

embedding distortion function and formulated the distortion minimization problem as a dynamic programming problem. A Viterbi algorithm is then used to find the minimum distortion LUT. Experiment results show that our presented scheme with a run constraint larger than 1 is more robust and has less distortion than traditional LUT embedding schemes such as odd-even LUT embedding. The presented embedding scheme is distinguished by its ability to achieve minimum distortion adaptively according to the distribution of the watermark signal. Future work may include exploring optimal LUT performances in the transform domain.

\section{REFERENCES}

[1] B. Chen and G.W. Wornell, "Quantization index modulation: A class of provably good methods for digital watermarking and information embedding," IEEE Trans. Inform. Theory, vol. 47, pp. 1423-1443, May 2001.

[2] I. Cox, J. Kilian, T. Leighton, and T. Shamoon, "Secure spread spectrum watermarking for multimedia," IEEE Trans. on Image Proc., vol. 6, pp. 1673-1687, Dec. 1997.

[3] M.M. Yeung and F. Mintzer, "An invisible watermarking technique for image verification," Proc. Int. Conf. Image Proc., vol. 2, pp. 680-683, 1997, Santa Barbara, CA.

[4] M. Wu, "Joint security and robustness enhancement for quantization based data embedding," IEEE Trans. on Cir. and Sys. for Video Tech., vol. 13, pp. 831-841, Aug. 2003.

[5] A.J. Viterbi and J.K. Omura, Principles of Digital Communication and Coding Processes, New York McGrawHill, 1979. 